\title{
SOLEIL, un centre de recherche pluridisciplinaire
}

\author{
Jean DAILLANT, Philippe DEBLAY, Frédérique FRAISSARD, Marie-Pauline GACOIN, Paul MORIN, Amor NADJI, Isabelle QUINKAL \\ Synchrotron SOLEIL, L'Orme des Merisiers, 91190 Gif-sur-Yvette \\ isabelle.quinkal@synchrotron-soleil.fr
}

Installation de très haute technologie, SOLEIL est à la fois une source de rayonnement d'une brillance exceptionnelle, une plateforme de service ouverte depuis 2008 à toutes les communautés scientifiques et industrielles, et un laboratoire de recherche à la pointe des techniques expérimentales.

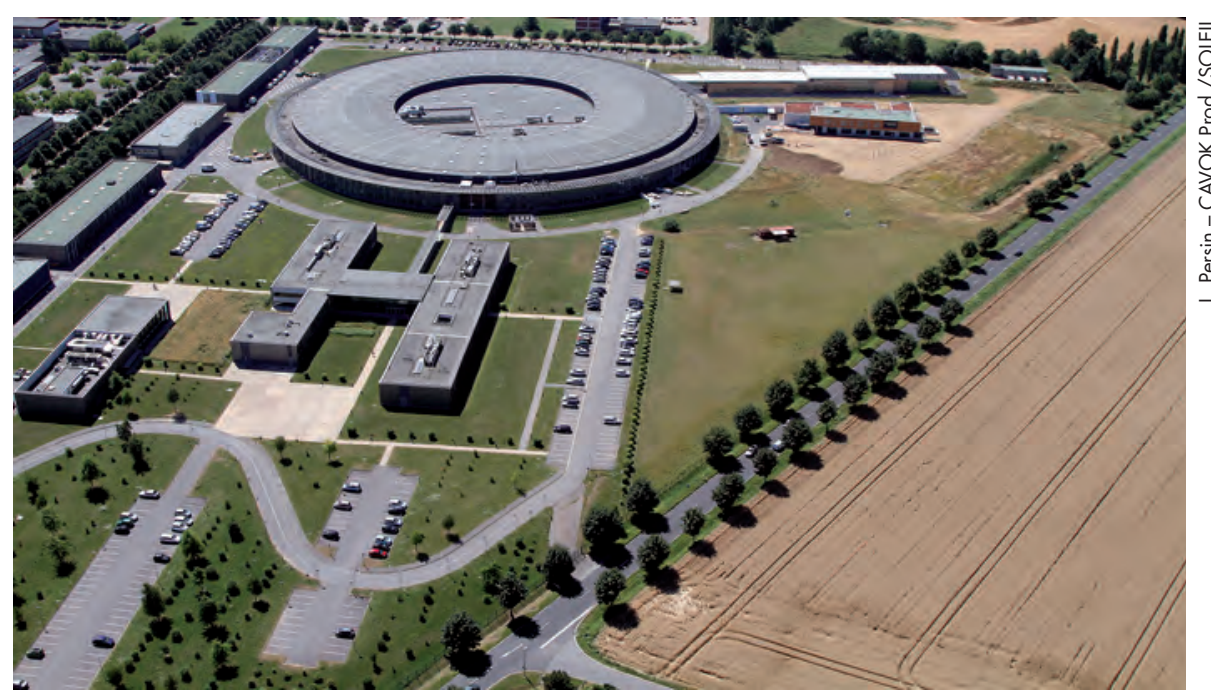

Figure 1. Les installations de SOLEIL sur le plateau de Saclay.

A jourd'hui 25 lignes de lumière - qui sont autant de laboratoires optimisés pour une technique ou une thématique particulière - fonctionnent en parallèle, nuit et jour, plus de 250 jours par an. D'ici 2016, quatre lignes supplémentaires viendront s'ajouter aux 25 existantes. Le personnel de SOLEIL accueille chaque année près de 4000 expériences de chercheurs venus de France et de l'étranger, et issus de tous les domaines qui aujourd'hui mobilisent la science et l'industrie.

\section{Un outil de recherche} $\approx$ aujourd'hui incontournable

Grâce à son large domaine spectral (de l'infrarouge aux rayons $X$ ), SOLEIL (figure 1) développe un très vaste programme scientifique permettant à la fois d'appréhender la structure et la géométrie de la matière à l'échelle atomique, et d'explorer les propriétés chimiques, électriques ou magnétiques de la matière sous toutes ses formes. Ces recherches sont menées via une large palette de techniques analytiques, qui fournissent des informations complémentaires intéressant aussi bien les chercheurs universitaires que les industriels. Quatre grandes familles de techniques sont ainsi mises en œuvre :

- diffraction/diffusion des rayons X: information structurale, au niveau moléculaire ou même atomique, des échantillons,

- spectroscopie d'absorption IR, UV et X : information chimique (nature et environnement d'un élément, degré d'oxydation...), - spectroscopie de photoémission d'électrons : propriétés électroniques et magnétiques,
- imagerie (plein champ et balayage) et radiographie:morphologie des échantillons.

- Grâce à ces techniques, plusieurs domaines en croissance importante occupent une place centrale dans les recherches menées à SOLEIL:

- la biologie et la pharmacie : recherche de nouveaux principes actifs, à partir de la visualisation à l'échelle atomique des interactions protéine-molécule candidate ;

- les matériaux : étude des nanomatériaux et des matériaux composites (matériaux de l'archéologie, de la paléontologie et du patrimoine culturel), contrôle de surface, vieillissement, durabilité et performances sous contraintes mécaniques ou thermiques ;

- la chimie : R\&D de nouveaux produits ou optimisation de fabrication, suivi des réactions chimiques en temps réel, détermination de la nature et de l'état d'oxydation des réactifs (développement de nouveaux catalyseurs) ;

- l'environnement et les éco-industries: analyse des matrices environnementales et des déchets, identification des micropolluants organiques, minéraux et radioactifs, spéciation des métaux lourds et santé environnementale (principalement autour des nanomatériaux) ;

- l'électronique : domaine des composants, de l'électronique professionnelle et industrielle, de l'optimisation du stockage de l'information ;

- l'énergie : étude des procédés énergétiques (batteries, cellules photovoltaïques, catalyseurs, biocarburants...); 
- les cosmétiques, pour le contrôle de l'efficacité et de l'innocuité des nouveaux produits ;

- et bien sûr des approches très fondamentales de compréhension à l'échelle atomique et moléculaire de l'interaction photon-matière.

\section{Des performances de haut niveau pour la machine}

L'opération de l'anneau de stockage avec les plus hautes performances reste la préoccupation collective des équipes du synchrotron, et la disponibilité du faisceau de photons pour les lignes de lumière atteint $98 \%$. Neuf des lignes ont pour source de lumière un dipôle, deux fonctionnent sur wiggler, et les 18 autres avec un ou plusieurs onduleurs, d'où la présence actuellement dans l'anneau de 26 éléments d'insertion de types très différents (voir en encadré le fonctionnement de SOLEIL). En octobre 2013 a été installé le « wiggler » qui fournira les photons pour la ligne PUMA, optimisée pour les matériaux anciens. Ce wiggler jouera par ailleurs le rôle de modulateur dans le projet «femto-slicing » qui permettra de générer des pulses femto-secondes de rayons $X$ à plus haute cadence combinant lasers femto-seconde (sur les lignes TEMPO, CRISTAL et, potentiellement, DEIMOS et GALAXIES) et rayonnement synchrotron, afin de développer des expériences résolues en temps.

D'autres éléments d'insertion sont en cours de conception ou de construction, le maintien au meilleur niveau des performances de SOLEIL passant par le développement d'insertions innovantes et une veille sur les sources de rayonnement utilisant des accélérateurs.

Dans ce cadre, des études sont en cours, notamment en collaboration avec le synchrotron suédois MAXIV pour un « wiggler sous-vide apériodique » et un « onduleur cryo-ready $»$.

SOLEIL est aussi impliqué dans des projets visant à développer les sources de lumière de demain. Associé à sept partenaires (ESRF, LAL, LOA, CEA (SPAM et SACM), LCPMR, PhLAM) reconnus comme les laboratoires experts du domaine en France, SOLEIL coordonne LUNEX5 (free electron laser using a new accelerator for the exploitation of $X$-ray radiation of 5 th generation) dont le but est de développer un dispositif de recherche à la fois plus compact et moins coûteux qui intégrera les tout derniers développements en matière d'accélération laser et permettra de lever des verrous scientifiques et technologiques auxquels sont confrontés tous les lasers à électrons libres actuels ou en construction.

Citons également le projet THOMX, source compacte de rayons $X$ à flux très élevé, dédiée à des applications médicales (imagerie statique et dynamique, mammographie en 3D, angiographie...) et aux sciences sociales (analyse des matériaux pour l'étude du patrimoine, datation des œuvres d'art...).

\section{Micro Spectromètre

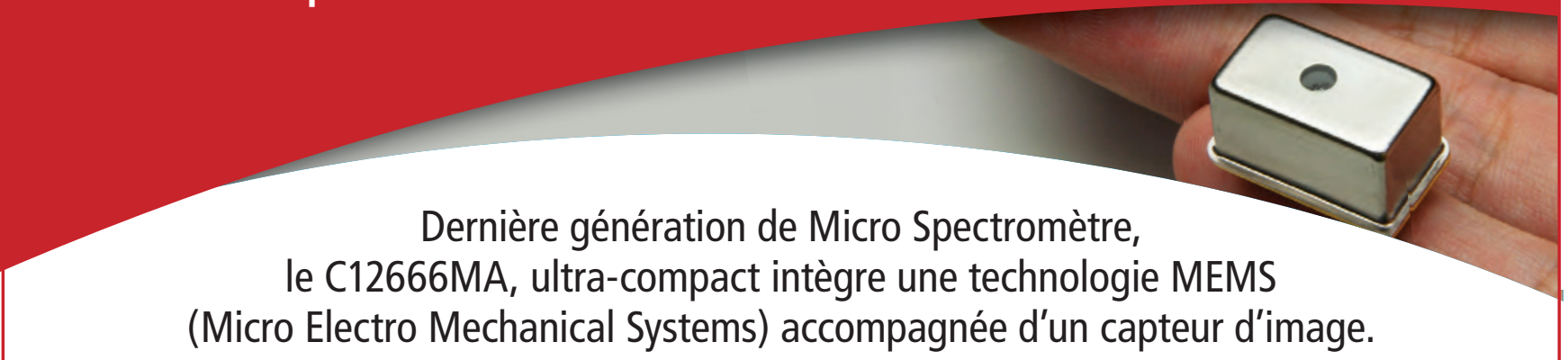

\section{Spécifications :}

- Taille : 20.1 x 12.5 x $10.1 \mathrm{~mm}$

- Boitier hermétique : haute fiabilité

- Faible consommation : idéal pour les appareils portables

- Poids : $5 \mathrm{~g}$

\section{Applications:}

- Contrôle de la qualité de l'eau

- Instruments de mesure pour l'environnement

- Contrôle de couleur

- Instruments de mesure connectés aux smartphones et tablettes

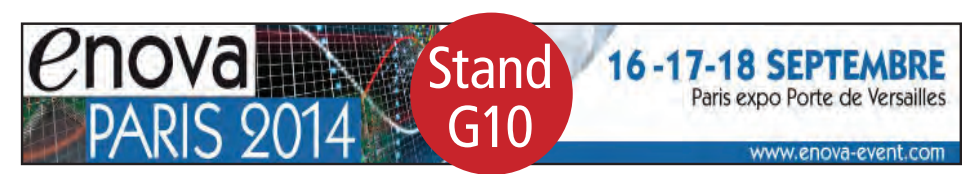

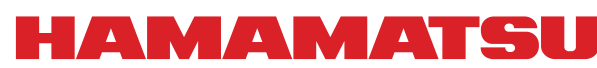

PHOTON IS OUR BUSINESS www.hamamatsu.fr - infos@hamamatsu.fr 


\section{Lignes de lumière \\ les dernières avancées}

Deux projets proposés et coordonnés par SOLEIL sont actuellement financés dans le cadre des «Investissements d'Avenir » (EquipEX). Le projet ROCK est centré sur la construction de la ligne de lumière du même nom, consacrée à l'étude des propriétés fonctionnelles des nanomatériaux utilisés en catalyse et dans les batteries. Elle accueillera ses premiers utilisateurs en février 2015. NANOIMAGESX finance un équipement unique en Europe pour l'imagerie 3D, en termes de sensibilité, de détection et de résolution spatiale: la ligne longue de nanotomographie ANATOMIX. Soutenue par des partenaires académiques et industriels dans les domaines du biomédical et des matériaux avancés, la ligne est en phase de conception avec des développements d'instruments originaux réalisés à SOLEIL.

Les lignes de lumière déjà fonctionnelles (figure 2) font également l'objet de toute l'attention des équipes de SOLEIL. La ligne MARS a pour objectif d'accroître les possibilités de recherche en physique, chimie et biologie sur la matière radioactive (émetteurs $\alpha, \beta$, $\gamma$ et neutrons). En septembre 2013, des expériences sur des échantillons radioactifs dépassant les seuils d'exemption y ont été réalisées par un groupe du CEA, suite à une première autorisation d'utilisation de la ligne au-dessus des seuils d'exemption, délivrée par l'Autorité de Sûreté Nationale fin 2012. Le passage aux étapes ultérieures, pour l'étude d'échantillons de plus forte radioactivité, est en cours.

Par ailleurs, la mise en place de systèmes d'acquisition à haut flux de données sur les lignes de lumière est un enjeu important pour SOLEIL. Le prototype du système FlyScan, acquisitions multi-détecteurs sur des systèmes en mouvement et cadencés par une électronique unique, a été enrichi et mis en œuvre sur la ligne METROLOGIE, donnant des résultats prometteurs. Son optimisation se poursuit en vue d'une utilisation sur NANOSCOPIUM et, dans un second temps, sur des lignes de lumière déjà ouvertes aux utilisateurs: sept lignes pourraient être concernées dans un premier temps suivies potentiellement de huit autres.

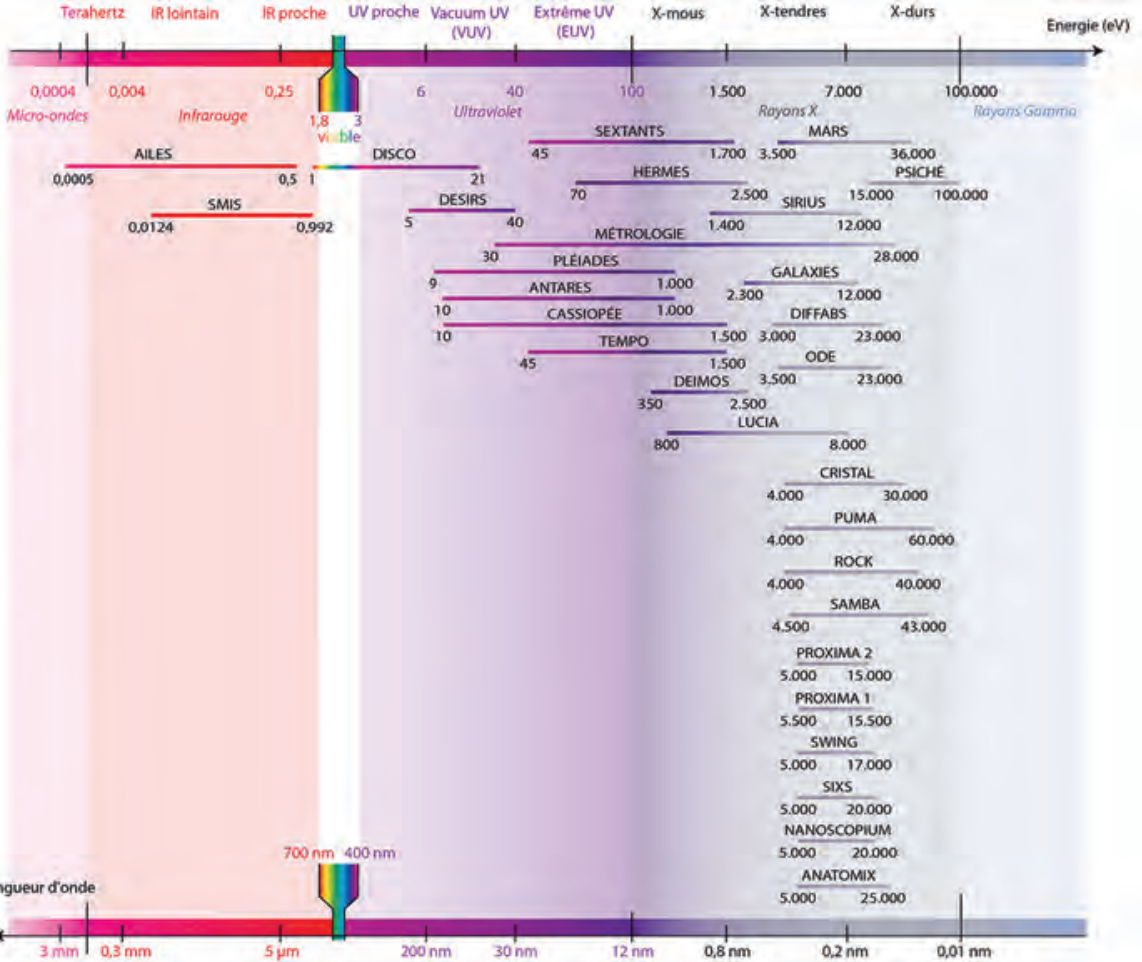

Figure 2. Les lignes de lumière de SOLElL classées selon leur gamme d'énergies/longueurs d'onde.

\section{Un nombre croissant d'utilisateurs}

En 2013, pour leur sixième année d'ouverture aux utilisateurs extérieurs, les lignes de lumière et les laboratoires de SOLEIL (Chimie, Surfaces et Biologie) ont compté près de 4000 visites - un même utilisateur pouvant venir plusieurs fois de collaborateurs de 690 laboratoires différents. Plus du quart de ces laboratoires sont étrangers, principalement européens (Royaume-Uni, Allemagne, Italie et Espagne sont les plus présents), les scientifiques en provenance des États-Unis venant par ailleurs depuis 2013 compléter le top five des communautés étrangères utilisatrices de SOLEIL.

Pour les accueillir, SOLEIL propose à ses utilisateurs quatre modalités d'accès complémentaires :

- Un accès gratuit après sélection par les pairs, « les Comités de Programmes ${ }^{1}$ » qui attribuent ainsi $65 \%$ du temps total de faisceau disponible pour les expériences dans le cadre de deux appels à projets annuels. Les quelque 600 projets scientifiques soumis à SOLEIL tous les 6 mois sont évalués et classés dans un cadre compétitif par 6 comités sur la base de leur qualité scientifique et de la pertinence d'utilisation du rayonnement synchrotron, avant soumission à la Direction Scientifique de SOLEIL pour validation. En moyenne sur l'ensemble des lignes de lumière, un projet sur deux est accepté. Les équipes sélectionnées ont l'obligation de soumettre un compte-rendu d'expérience et de publier leurs résultats, avec mention de l'utilisation de SOLEIL.

- Si les études synchrotron nécessitent de la confidentialité, les utilisateurs externes ont recours aux prestations payantes, «à la carte »: de la simple mise à disposition d'une ligne de lumière jusqu'à la prestation complète : préparation des échantillons, collecte, analyse et interprétation des données, rédaction du rapport d'analyse.

\footnotetext{
Intitulés des Comités : 1. Matière diluée ; 2. Propriétés électroniques et magnétiques de la matière - Surfaces et Interfaces ; 3. Propriétés de la matière et des matériaux; 4 . Chimie et physico-chimie - Réactivité in situ - Matière molle 5. Biologie - Santé ; 6. Matériaux anciens - Terre et Environnement.
} 
L'accès à SOLEIL se fait alors sans sélection préalable, sur des périodes correspondant à $10 \%$ du temps total de faisceau disponible, et le niveau de confidentialité demandé est garanti à l'utilisateur.

- Dans le cadre de l'activité de recherche scientifique et développement instrumental menée par le personnel de SOLEIL, 20\% du temps total de faisceau disponible est dédié à des actions de recherche partenariale avec des équipes externes, publiques et/ou privées. La règle du partage est appliquée dans un cadre contractuel : mise en commun de moyens (intellectuels, humains, techniques, financiers), définition des règles de partage de la propriété et des modalités d'exploitation des résultats de la recherche.

- Enfin, un nombre très limité de projets peut être accepté pour des travaux urgents, pendant les $5 \%$ du temps total de faisceau restants.

\section{La science ensemble}

SOLEIL considère comme prioritaire et fertile l'ouverture du synchrotron à la société et aux partenariats de toute forme, et a à cœur de mettre la science au cœur de la société, au travers de nombreuses actions de médiation scientifique. Ses équipes vont à la rencontre du public lors d'événements extérieurs classiques tels que la Fête de la Science, les Années Internationales, les Journées du Patrimoine ou la Nuit des Chercheurs. SOLEIL accueille également près de 5000 visiteurs chaque année, de tous horizons, et leur propose des programmes et des outils pédagogiques documentaires et expérimentaux. Une ouverture quotidienne depuis sa conception, qui consolide l'importance pour SOLEIL de partager les connaissances au fur et à mesure qu'elles évoluent.

\section{Comment fonctionne SOLEIL}

Les expériences menées dans un synchrotron utilisent le rayonnement électromagnétique émis par des particules chargées, de très haute énergie. À SOLEIL ce sont des électrons, accélérés quasiment à la vitesse de la lumière dans un premier accélérateur, linéaire (le LINAC), d'une longueur de $16 \mathrm{~m}$, dont le rôle est de fabriquer un faisceau d'électrons pulsé (des bouffées de 300 ns, trois fois par seconde) d'une énergie de $100 \mathrm{MeV}$. À la sortie du LINAC, les électrons entrent dans le Booster, synchrotron de $157 \mathrm{~m}$ de périmètre. En une fraction de seconde, leur énergie passe de $100 \mathrm{MeV}$ à $2750 \mathrm{MeV}(2,75 \mathrm{GeV})$ par une accélération due à un champ électrique généré par une cavité radiofréquence $(352 \mathrm{MHz})$. Pendant cette montée en énergie, les dimensions transverses du faisceau sont comprimées.

Une fois que les électrons ont atteint l'énergie de 2,75 GeV, ils sont extraits du Booster pour être envoyés vers I'anneau de stockage, où ils tournent pendant des heures. Les électrons circulent dans un tube d'environ $5 \mathrm{~cm}$ de diamètre où règne un vide très poussé $\left(10^{-9} \mathrm{mbar}\right)$, afin de minimiser les collisions qu'ils pourraient avoir avec les atomes de gaz résiduels. L'anneau de stockage, de 354 mètres de périmètre, est constitué d'une succession :

- de virages où se trouvent les éléments magnétiques de courbure (appelés aussi aimants de courbure ou dipôles),

- de parties rectilignes où se trouvent les éléments magnétiques de focalisation (quadrupôles et sextupôles) qui permettent de réduire la taille du faisceau à 4 dixièmes de $\mathrm{mm}$ de large et 2 centièmes de $\mathrm{mm}$ de haut (soit l'épaisseur d'un cheveu),

- de sections droites où sont placés des éléments magnétiques d'insertion (wiggler et onduleurs) qui, en faisant osciller la trajectoire du faisceau d'électrons, produisent du rayonnement synchrotron.

Chaque fois que les électrons se déplacent de façon non rectiligne et non uniforme - ce qui est le cas dans les dipôles et les éléments d'insertion - ils subissent une accélération et perdent de l'énergie sous forme de rayonnement synchrotron. Celui-ci est recueilli, conditionné et utilisé tout autour de l'anneau dans les lignes de lumière, où se déroulent les expériences.

Les lignes de lumière sont de véritables laboratoires instrumentés pour préparer et analyser les échantillons à étudier, et traiter les informations recueillies. Toutes les expériences sont basées sur l'analyse de l'interaction entre échantillon et rayonnement synchrotron. Celui-ci possède des caractéristiques uniques de polarisation, $d^{\prime}$ accordabilité sur une très large gamme d'énergie de photons (de l'infrarouge aux rayons $X$ durs), de brillance, ainsi qu'une structure temporelle. Autant de propriétés mises à profit par exemple pour étudier la matière ultra-diluée, suivre des processus à l'échelle de quelques centièmes de nanoseconde, ou obtenir des données à des échelles de taille de l'ordre de quelques dizaines de nanomètres.

Les 29 lignes de SOLEIL sont complémentaires les unes des autres et chacune d'entre elles est orientée pour des techniques, des domaines de recherche et des applications particulières.

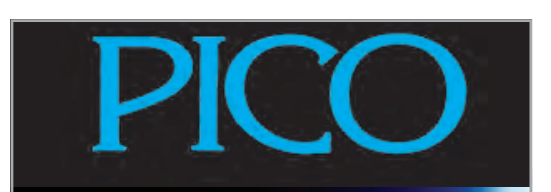

DC-DC CONVERTERS $2 V$ to 10,000 VDC Outputis 1-300 Watt Modules

Regulated/Proportional/Programmab]e Isolated/Single/Dual Outputs

High Reliability

Greater than $1,000,000$ hirs Mil Hbk 217F

Military Upgrades

Expanded operating temperature $-55^{\circ}$ to $+85^{\circ} \mathrm{C}$, no derating required

Environmental Screening

Selected Screening, Mil Std 883

ULTRA Miniature From $0.5^{\prime \prime} \times 0.5^{\prime \prime} \times 0.2^{\prime \prime}$

Surface Mount/Thru-Hole

- Custom Models

- US Manufactured

- AS 9100 Approved

High Reliability AC/DC

Power Supplies, Transformers and Inductors

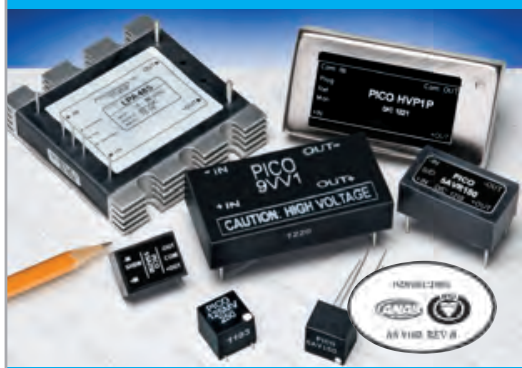

See PICO's full line catalog at www.picoelectronics.com

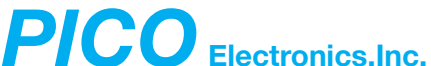

143 Sparks Ave. Pel ham N. Y. 10803

E Mail: info@picoelectronics.com

Pico Representatives

France

ELCOS BVBA

E-mail: lbarbe@elcos.be

Phone: 003232182073

Germany

ELBV/Electronische Bauelemente Vertrieb E-mail: info@elbv.de Phone: 0049894602852 England

Ginsbury Electronics Ltd.

E-mail: rbennett@ginsbury.co.uk Phone: 00441634298900 